\title{
Glutamine protects the small intestinal mucosa in anticancer drug-induced rat enteritis model
}

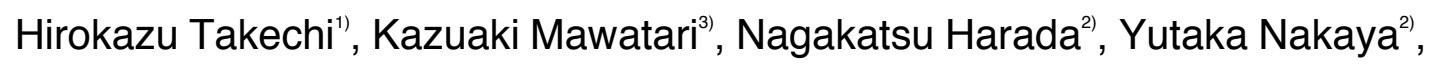 \\ Megumi Asakura ${ }^{2)}$, Mutsumi Aihara ${ }^{3)}$, Hiromitsu Takizawa", Masakazu Goto", \\ Takeshi Nishino", Takuya Minato", Yoshihito Furukita", Yota Yamamoto", \\ Yasuhiro Yuasa", Hiromichi Yamai", Takahiro Yoshida"), Junichi Seike"), and \\ Akira Tangoku' \\ 1)Department of Thoracic, Endocrine Surgery and Oncology, Institute of Health Biosciences, the Uni- \\ versity of Tokushima Graduate School, Tokushima, Japan, ${ }^{2}$ Department of Nutrition and Metabolism, \\ Institute of Health Biosciences, the University of Tokushima Graduate School, Tokushima, Japan, \\ ${ }^{3}$ Department of Preventive Environent and Nutrition, Institute of Health Biosciences, the University \\ of Tokushima Graduate School, Tokushima, Japan
}

\begin{abstract}
Supportive therapy during chemotherapy has become essential, but effective preventive therapies to gastrointestinal mucosal injury are few. We investigated the efficacy of glutamine in rat anticancer drug-induced enteritis model. In this study, we used twenty male SD rats. They were divided into control, 5 -fluorouracil (5-FU) (orally administered at $20 \mathrm{mg} / \mathrm{kg}$ day $), 5-F U+$ glutamine $(1000 \mathrm{mg} / \mathrm{kg} / \mathrm{day})$ and $5-\mathrm{FU}+\mathrm{glutamine}+$ fiber and oligosaccharide $\left(\mathrm{GFO}^{\circledR}\right)(1000 \mathrm{mg} / \mathrm{kg} / \mathrm{day})$ groups. All groups were sacrificed on day 6 and upper jejunums were excised. The jejunal villous height was measured in specimens. IgA level in jejunal washing solution, and serum diamine oxidase activity were also measured. The jejunal villous height was recognized as shorter in the specimen from 5 -FU treated rats compared with $5-\mathrm{FU}+$ glutamine treated rats $(\mathbf{p}<0.001)$. Serum diamine oxidase activity in 5-FU+glutamine group were significantly superior to that in 5-FU group ( $p=0.028$ ). IgA level in jejunal washing solution tended to be higher in 5 -FU+glutamine group than that in 5 -FU group $(p=0.076)$. On the other hand, serum diamine oxidase activity and IgA level in jejunal washing solution showed no significant difference between 5-FU+GFO and 5-FU treatment group. Our results suggest that glutamine showed protective effects on mucosal injury of small intestine in rat anticancer drug-induced enteritis model. J. Med. Invest. 61 : 59-64, February, 2014
\end{abstract}

Keywords : glutamine, gastrointestinal toxity, anticancer drug, diamine oxidase, IgA

Received for publication October 7, 2013 ; accepted November $19,2013$.

Address correspondence and reprint requests to Akira Tangoku $\mathrm{MD}, \mathrm{PhD}$, Department of Thoracic, Endocrine Surgery and Oncology, Institute of Health Biosciences, the University of Tokushima Graduate School, 3-18-15 Kuramoto, Tokushimacity, Tokushima, 770-8503, Japan and Fax : +81-88-633-7144.

\section{INTRODUCTION}

The role of chemotherapy has been more important in recent multidisciplinary cancer therapy. To improve the therapeutic effects, a countermeasures against adverse events and a supportive therapy become very important. Adverse events in digestive 
system like nausea, vomiting and diarrhea frequently occur and interrupt the continuation of treatment. Nausea, vomiting has become controllable due to the improvement of antiemetic drugs, but effective preventive therapy is lacking for diarrhea. Atrophy of the small intestinal mucosa (SIM) might induce sepsis due to bacterial translocation (BT). In this pathological condition, declines of not only the intestinal barrier system but also an immune dysfunction occur. Lymphocytes in the lamina propria mucosa are important cellular mediators of immunity in SIM. $\mathrm{CD} 4+\mathrm{T}$ cells are surrounded by abundant IgA-secreting cells, and mainly engaged in humoral immunity (1-4). When the mucosa is injured, the number and activity of these lymphocytes decrease and aggravate the immune condition.

L-glutamine (glutamine) is an amino acid abundant in the body, and plays an important role, as an energy source for cells with a fast metabolic turnover. Particularly, it has been reported that glutamine is the major energy substrate of SIM (5).

The preventing effect of glutamine for atrophy of SIM in an animal enteritis model $(6,7)$, in animal parenteral nutrition model (8), and in animal intestinal ischemia-reperfusion injury model (9), has been reported, but there were no reports concerning the effects of glutamine for protection of SIM and IgA secretion in the condition of anticancer drug-induced enteritis. In this study, the preventing effect of glutamine for atrophy of SIM was investigated with regard to the histological and immunological view in rat anticancer drug-induced enteritis model.

\section{MATERIAL AND METHODS}

Animals : Seven-week-old male SD rats (Japan SLC Inc, Hamamatsu, Japan) were used. The animals were acclimated for 7 days, and given free access to food (MF : Oriental Yeast Industry, Tokyo, Japan) and drinking water. The experiment was performed following the animal management regulations of Tokushima University.

Drugs : 5-FU (Wako Pure Chemical Industries, Osaka, Japan) was orally administered at $20 \mathrm{mg} /$ $\mathrm{kg} /$ day. The drug was suspended and adjusted to 2 $\mathrm{mg} / \mathrm{mL}$ with $0.5 \%$ hydroxypropyl methyl cellulose (HPMC : Shinetsu Chemical, Tokyo, Japan) (10).

Supplements : Glutamine (Wako Pure Chemical Industries, Osaka, Japan) was orally administered at $1,000 \mathrm{mg} / \mathrm{kg} /$ day. In addition, GFO (Otsuka Pharmaceutical, Tokyo, Japan), containing ingredients such as soluble indigestible dietary fiber and oligosaccharides (Table) which was expected to promote growth of SIM, was administered orally at 1,000 $\mathrm{mg} / \mathrm{kg} /$ day $(200 \mathrm{mg} / \mathrm{kg} /$ day as glutamine). Both supplements were suspended and adjusted to 100 $\mathrm{mg} / \mathrm{mL}$ with $0.5 \%$ HPMC (10). We determined the dose of glutamine and GFO by reference to the past report (10) and preliminary experimental results.

Administration of 5-FU and supplements : Twenty rats were allocated to each of the following 4 groups (5 rats in each) : Control group, 5-FU group, 5-FU+ glutamine group, 5 -FU+GFO group : Glutamine and GFO were administered to Groups 3 and 4, from 3 days before the study. On day 1-5, 5-FU was administered to Group 2-4. In addition, glutamine was administered to Group 3 and GFO to Group 4, four hours before 5 -FU administration. We administered 5-FU and supplements to all rats orally using dropsonde into the esophagus of rat. All groups were sacrificed under deep ether anesthesia on day 6 .

\begin{tabular}{lcc} 
Table. & Nutritional factors of $\mathrm{GFO}^{\circledR}(15 \mathrm{~g})$ \\
\hline calorie & & $36 \mathrm{kcal}$ \\
protein & (glutamine) & $3.6 \mathrm{~g}(3.0 \mathrm{~g})$ \\
fat & & $0 \mathrm{~g}$ \\
carbohydrate & (lactosucrose) & $6.01 \mathrm{~g}(1.45 \mathrm{~g})$ \\
dietary fiber & $5.0 \mathrm{~g}$ \\
natrium & & $0.2 \sim 1.2 \mathrm{mg}$ \\
\hline
\end{tabular}

\section{MEASUREMENT}

(1) Calorie intake and body weight: During the study, the dietary calorie intake was measured daily from day 1 to 5 , and the body weight was measured daily from day 1 to 6 .

(2) IgA level in jejunal washing solution : The jejunum was excised for histological examination, and the lumen was washed with $20 \mathrm{~mL}$ of PBS. The IgA level in the washing solution was measured by ELISA using a RAT IgA QUANTITATION KIT (BETHYL Laboratories, INC, Montgomery, USA). (3) Jejunal villous height: The jejunum was fixed in $10 \%$ neutral formalin, and paraffin-embedded, and $5-\mu \mathrm{m}$ thin sections were prepared using a microtome. After being deparaffinized, the sections were stained with hematoxylin-eosin, and the villi $(\mu \mathrm{m})$ in the 10 sections were measured with 100times magnification microscope.

(4) Serum diamine oxidase (DAO) activity : Blood 
(5 ml) was collected from the abdominal aorta. After standing at room temperature for about 30 minutes, the blood sample was centrifuged $\left(3,000 \mathrm{rpm}\right.$ at $4^{\circ} \mathrm{C}$ for 15 minutes), and $500 \mu 1$ of serum was collected. The samples were stored at $-80^{\circ} \mathrm{C}$. The DAO activity was measured following Takagi's method (11) : Cadaverine solution and serum were combined and heated, followed by the addition of a color former containing DA-67 and peroxidase, and the activity was measured by colorimetry at $668 \mathrm{~nm}$ using a spectrophotometer.

\section{STATISTICAL ANALYSIS}

Data were expressed Mean \pm SD. For comparison of the change of calorie intake and body weight, serum diamine oxidase activity, IgA level in the jejunal washing solution and the jejunal villous height, Student's t-test was used. A value of $p<0.05$ was regarded as a significant.

\section{RESULTS}

(1) Changes in the calorie intake and body weight

The calorie intake was decreased in 5-FU+glutamine group on day 4 and 5 compared to that in control group. The intake was also decreased in the 5 -FU and 5 -FU+GFO groups, and the decrease on day 5 was marked in both groups (Fig. 1). The body weight continuously increased in control group, reaching an $8.2 \%$ increase on day 6 compared to that on day 1 . In contrast, body weight decreased over the period in 5 -FU group, and the total decrease on the final day was $11.7 \%$. A $2.8 \%$ increase was noted in 5-FU+glutamine group, but a 5.5\% decrease was noted in 5-FU+GFO group (Fig. 2).

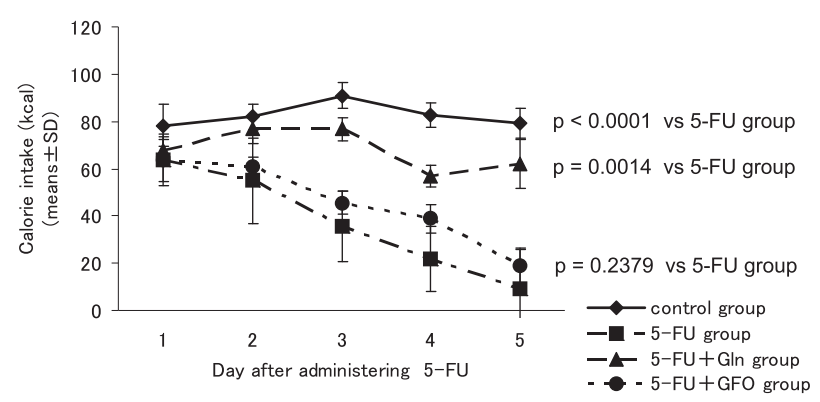

Fig. 1. Calorie intake in each group : The calorie intake decreased in $5-\mathrm{FU}+$ glutamine group on day 4 and 5 . The calorie intake decreased throughout the period in 5 -FU and $5-\mathrm{FU}+\mathrm{GFO}$ group.

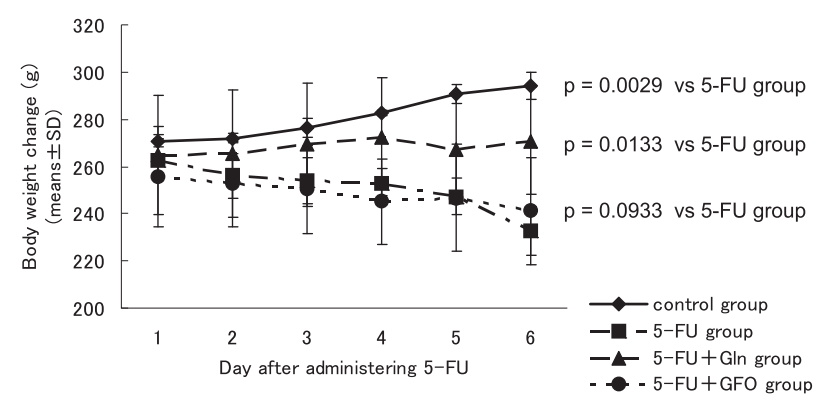

Fig. 2. Body weight in each group : The body weight decreased over the period in 5-FU group, and the total decrease on day 6 was $11.7 \%$. A $2.8 \%$ increase was noted in 5 -FU + glutamine group, but a $5.5 \%$ decrease was noted in $5-\mathrm{FU}+\mathrm{GFO}$ group.

(2) IgA level in jejunal washing solution

Compared to $21,685.1 \pm 9,059.2 \mathrm{ng} / \mathrm{mL}$ in control group, the IgA level was markedly decreased $(210.3 \pm 48.6 \mathrm{ng} / \mathrm{mL})$ in 5 -FU group, suggesting a marked decline of immunity $(\mathrm{p}=0.033)$. The level was $6,578.3 \pm 5,708.8 \mathrm{ng} / \mathrm{mL}$ in 5 -FU+glutamine group, showing a higher level than that in 5 -FU group, but difference was not significant $(\mathrm{p}=0.150)$. In 5-FU+GFO group, the level was 2,280.7 $\pm 1,345.6$ $\mathrm{ng} / \mathrm{mL}$, showing no significant difference from that in 5-FU group ( $\mathrm{p}=0.870)$ (Fig. 3).

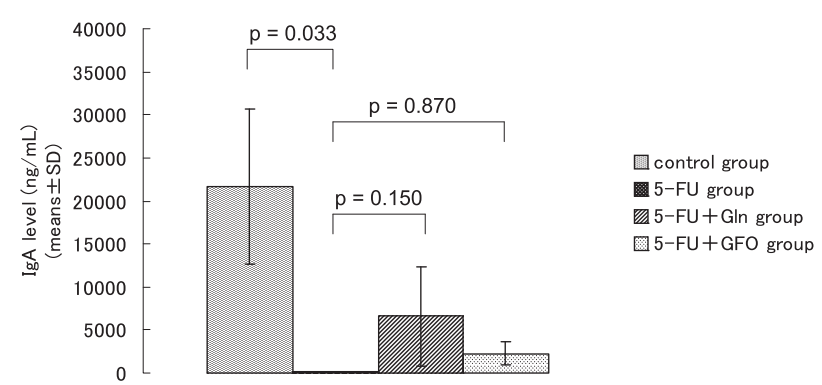

Fig. 3. Secretory IgA levels of jejunal washing solution : The IgA level was markedly decreased in 5 -FU group compared to that in control group. In 5-FU+glutamine group, the level was higher than that in 5-FU group, but the difference was not significant. In 5-FU+GFO group, the level shows no significant difference from that in 5-FU group.

\section{(3) Jejunal villous height}

The jejunal villous height was $445.3 \pm 12.9 \mu \mathrm{m}$ in control group, whereas it was significantly shortened to $223.9 \pm 12.4 \mu \mathrm{m}$ in 5-FU group $(\mathrm{p}<0.001)$. In contrast, the height was $334.6 \pm 19.7 \mu \mathrm{m}$ in 5 $\mathrm{FU}+$ glutamine group, showing that the villi were significantly higher than those in 5-FU group ( $\mathrm{p}<$ 0.001 ). In $5-\mathrm{FU}+\mathrm{GFO}$ group, height was $236.34 \pm$ $9.45 \mu \mathrm{m}$, showing significant shortening compared 
to that in $5-\mathrm{FU}+$ glutamine group $(\mathrm{p}<0.001)$, but significantly higher than that in 5 -FU group $(\mathrm{p}=0.027)$ (Figs. 4 and 5).

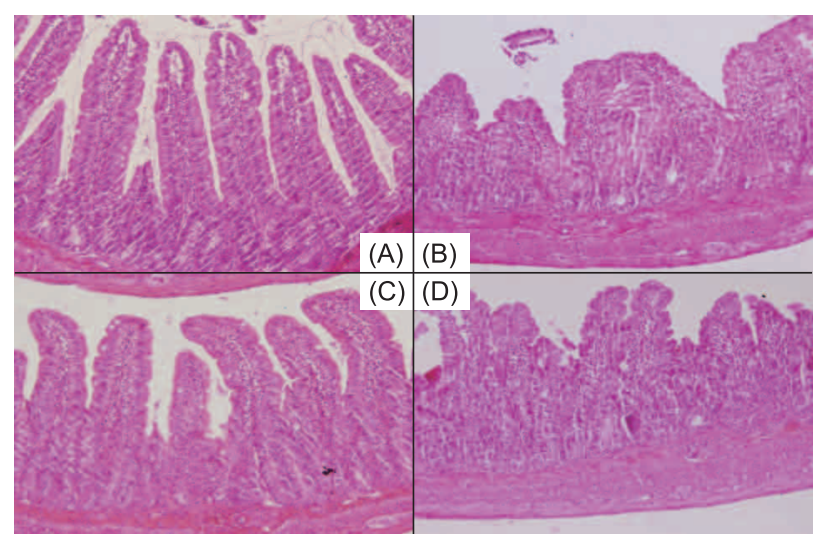

Fig. 4. Specimens of the jejunal villi $(\times 100)$ : (A) control group : (B) 5-FU group : (C) 5-FU+glutamine group : (D) 5-FU+GFO group : Marked atrophy of villi was seen in 5-FU group and 5$\mathrm{FU}+\mathrm{GFO}$ group compared to that in control group. In 5-FU+ glutamine group, the morphology of vill was maintained well compared to that in 5 -FU group and 5 -FU+GFO group.

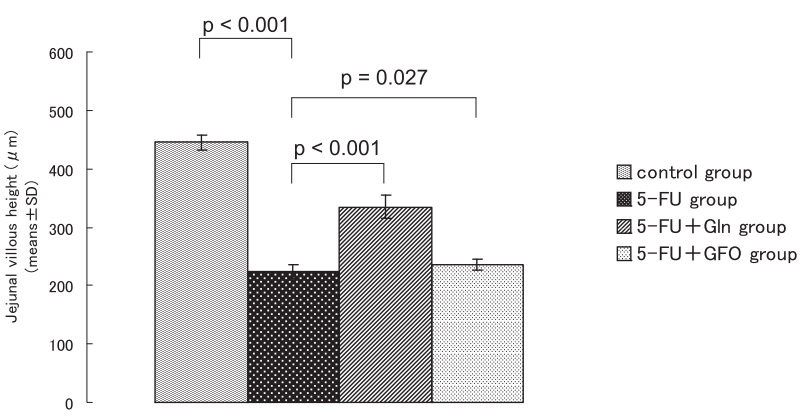

Fig. 5. Jejunal villous height: The jejunal villous height was significantly shorter in 5-FU group than those in control group. In 5-FU+glutamine group, it was significantly higher than those in 5-FU group. In 5-FU+GFO group, it was shorter than that in 5 -FU+glutamine group (data not shown), but higher than that in 5 -FU group.

\section{(4) Serum DAO activity}

Compared to $6.82 \pm 1.6 \mathrm{U} / \mathrm{L}$ in control group, the activity level $(3.45 \pm 1.22 \mathrm{U} / \mathrm{L})$ was significantly decreased in 5 -FU group $(p=0.0024)$. In 5 - FU+glutamine group, the activity level was $5.43 \pm 0.65 \mathrm{U} / \mathrm{L}$, showing a significant inhibition of activity reduction compared to that in 5-FU group $(\mathrm{p}=0.0072)$. In contrast, no significant inhibition of activity reduction $(4.02 \pm 1.8 \mathrm{U} / \mathrm{L})$ was noted in $5-\mathrm{FU}+\mathrm{GFO}$ group $(\mathrm{p}=0.879)$, showing consistency with the histological findings, respectively (Fig. 6).

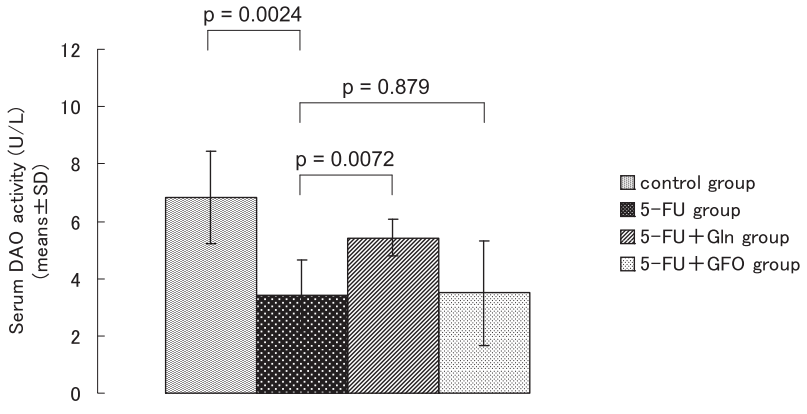

Fig. 6. Plasma diamine oxidase (DAO) activity : The plasma DAO activity level was significantly decreased in 5-FU group compared to that in control group. In 5-FU+glutamine group, it was significantly higher than that in 5-FU group. In contrast, it showed no significant difference between $5-\mathrm{FU}+\mathrm{GFO}$ group and 5-FU group.

\section{DISCUSSION}

The purpose of this study was to find effective preventing therapy for anticancer drug induced diarrhea. 5-FU was selected as an anticancer drug because it was very popular effective drug for many kind of cancers and it has been reported induces intestinal mucosal injury in rat model. Enteritis model was made by administering 5 -FU to rats orally at 20 $\mathrm{mg} / \mathrm{kg}$ once daily for 5 days as reported before (10).

The dietary calorie intake was maintained, and weight loss was inhibited throughout the period in 5 -FU+glutamine group compared to those in 5 -FU group. Histologically, the jejunal mucosal villous height was maintained, suggesting a protective effect of glutamine on SIM injury, as previously reported $(6,7)$.

We also focused on DAO activity, which is considered to be an index of SIM condition. DAO is a polyamine-degrading enzyme localized in the small intestine and placenta, and controls growth of SIM in which cell turnovers are very rapid $(12,13)$. A correlation between DAO activity levels in small intestinal tissue and the blood has been reported, based on which it is considered that the SIM condition can be objectively measured by serum DAO activity (14). DAO activity was also maintained by glutamine treatment. Therefore an efficacy of glutamine on anticancer drug-induced enteritis was suggested .

A total of 200 billion immunocytes are present in the human small intestine. $60-70 \%$ of all peripheral lymphocytes concentrate in the intestine, therefore the intestine was called the largest immune organ in the body (15). The intestinal mucosal immune system is roughly divided into gut-associated lymphoid 
tissue, such as Peyer's patches and mesenteric lymph nodes, and lymphocytes functioning as cellular mediators of immune system. When antigens and pathogens are incorporated by Microfold cell in Peyer's patch, initiating an immune response, IgApositive B cells are formed in the follicular center. These cells enter the lymph flow and then the systemic circulation through the mesenteric lymph nodes and thoracic duct, and finally reach the lamina propria mucosae of the airway, intestine, and vagina. This pathway is termed "the common mucosal immune system". The IgA-positive B cells which reach the lamina propria mucosae through this system are influenced by IL- 4 and IL- 5 produced by CD $4+\mathrm{T}$ cells, differentiated and matured into plasma cells, and produced IgA, exerting humoral immunity (16, 17). Therefore we measured the IgA level in the jejunal washing solution from an immunological aspect. It was decreased in 5-FU+glutamine group compared to that in control group, but the decrease was smaller than that in 5-FU group $(\mathrm{p}=0.076)$.

Since glutamine is an energy source for rapidly growing cells, it may also be used as an energy source by cancer cells. It should be considered that cancer cell's growth might be accelerated by glutamine. In a study using methotrexate-treated rats that were transplanted fibrosarcoma cells, Klimberg et al. compared normal diet group and glutamineadded diet group, and they observed not only the reduction of the gastrointestinal mucosal disorder, but also the decreasing of the mortality in glutamineadded diet group. They concluded that the addition of glutamine supported the anticancer effect of methotrexate $(10,18)$.

Regarding the route of administration, it is apparent that enteral administration is the most efficient supply route based on the fact that an energy for chorioepithelial cells actively synthesizing protein is mostly supplied through the intestine $(6,19-21)$.

Prebiotics, such as dietary fibers and oligosaccharides, are fermented by the large intestinal flora, and short-chain fatty acids are produced. As reported before, these short-chain fatty acids might increase enteroglucagon secretion from the ileum and colon, and might promote growth of SIM (22). We used GFO as a supplement candidate for promoting of SIM, but it did not improve the condition in 5-FU induced enteritis model. It might be due to a negative influence of mucosal stimulation by indigestible substances. Although GFO was administered from 3 days earlier than 5-FU administration, no benefical effects were noted in all rats, and diarrhea was worsened in some GFO treated rats.

In conclusion, we found glutamine played an important protective role for anticancer drug-induced small intestinal mucosa injury in rats by preventing mucosal atrophy and induced immunity. Glutamine might also play an important role in clinical practice.

\section{REFERENCES}

1. Kamata T, Nogaki F, Fagarasan S, Sakiyama T, Kobayashi I, Miyawaki S, Ikuta K, Muso E, Yoshida H, Sasayama S, Honjo T: High frequency of surface IgA positive plasma cells in the intestinal lamina propria of HIGA mice, a murine model IgA nephropathy with Hyperserum IgA. J Immunol 165 : 1387-1394, 2000

2. Zeitz M, Quinn TC, Graeff AS, James SP : Mucosal T cells provide helper function but do not proliferate when stimulated by specific antigen in lymphogranuloma venereum proctitis in nonhuman primates. Gastroenterology 94 : 353-366, 1988

3. Taguchi T, McGhee JR, Coffman RL, Beaqley KW, Eldridge JH, Takatsu K, Kiyono H : Analysis of Th1 and Th2 cells in murine gut-associated tissues. Frequencies of CD4+and CD8+T cells that secrete IFN- $\gamma$ and IL-5. J Immunol $145: 68-77,1990$

4. Gonnella PA, Chen Y, Inobe J, Komagata Y, Quartulli M, Weiner HL: In situ immune response in gut-associated lymphoid tissue (GALT) following oralantigen in TCR transgenic mice. J Immunol 160 : 4708-4718, 1998

5. Windmueller HG, Spaeth AE : Uptake and metabolism of plasma glutamine by the small intestine. J Biol Chem 249 : 5070-5079, 1974

6. O'Dwyer ST, Smith RJ, Hwang TL, Wilmore DW : Maintenance of small bowel mucosa with glutamine-enriched parenteral nutrition. JPEN $13:$ 579-585, 1989

7. Grant JP, Snyder PJ : Use of L-glutamine in total parenteral nutrition. J Surg Res 44 : 506-511, 1988

8. Burke DJ, Alverdy JC, Aoys E, Moss GS : Glutamine supplemented TPN improves gut immune function. Arch Surg 124 : 1396-1399, 1989

9. Alverdy JA, Aoys E, Weiss-Carrington P, Burke DA : The effect of glutamine-enriched TPN on gut immune cellularity. J Surg Res $52: 34-38$, 1992 
10. Tanaka A, Takeuchi K : Prophylactic Effect of L-Glutamine against Intestinal Derangement Induced 5-Fluorouracil or Indomethacin in Rats. Jpn Pharmacol Ther 30 : 455-462, 2002

11. Takagi K, Nakao M, Ogura Y, Nabeshima T, Kunii A : Sensitive colorimetric assay of serum diamineoxidase. Clinica Chimica Acta 226 : 67 75, 1994

12. Wolvekamp MC, de Bruin RW : Diamine Oxidase : An overview of historical, biochemical and functional aspects. Dig Dis $12: 2-14,1994$

13. Mennigen R, Bieganski T, Elbers A, Kusche $\mathrm{J}$ : The histamine-diamine oxidase system and mucosal proliferation under the influence of aminoguanidine and seventy percent resection of the rat small intestine. Agents Actions 27 : 221-223, 1989

14. Luk GD, Bayless TM, Baylin SB : Plasma postheparin diamine oxidase. Sensitive Provocative test for quantitating length of acute intestinal mucosal injury in the rat. J Clin Invest 71 : 1308-1315, 1983

15. Macpherson AJ, Gatto D, Sainsbury E, Harriman GR, Hengartner H, Zinkernagel RM : A primitive T cell-independent mechanism of intestinal mucosal IgA responses to commensal bacteria. Science $288: 2222-2226,2000$
16. Kudsk KA : Current aspects of mucosal immunology and its influence by nutrition. Am J Surg 183 : 390-398, 2002

17. Kudsk KA, Li J, Renegar KB : Loss of upper respiratory tract immunity with parenteral feeding. Ann Surg 223 : 629-635,1996

18. Klimberg VS, Nwokedi E, Hutchins LF, Pappas AA, Lanq NP, Broadwater JR, Read RC, Westbrook KC : Glutamine facilitates chemotherapy while reducing toxicity. J parenter Enteral Nutr 16 : 83S-87S, 1992

19. Karner J, Roth E, Ollenschlager G, Furst P, Simmei A, Karner J : Glutamine-containing dipeptides as infusion substrates in the septic state. Surgery 106 : 893-900, 1989

20. Souba WW, Smith RJ, Wilmore DW : Glutamine metabolism by the intestinal tract. JPEN $9: 608-617,1985$

21. Higashiguchi T, Hasselgren PO, Wagner K, Fischer JE : Effect of glutamine on protein synthesis in isolated intestinal epithelial cells. JPEN $17: 307-314,1993$

22. Breuer R, Buto SK, Christ ML, Bean J, Vernia P, Di Paolo MC, Caprilli R: Rectal irrigation with short-chain fatty acids for distal ulcerative colitis. Dig Dis Sci 36 : 185-187, 1991 